\title{
The Analysis of Music Therapy in Children with Autism Treatment based on Computer Technology
}

\author{
Liu Yan ${ }^{1}$,Wan Li-lii ${ }^{1}$,Wu Ya-fen ${ }^{2}$ \\ (1. College of Humanities, Jiangxi University of Traditional Chinese Medicine, Nanchang Jiangxi \\ 330004, China \\ (2. Scientific Research Center,Jiangxi University of Traditional Chinese Medicine, Nanchang \\ Jiangxi 330004, China
}

Keywords: Computer technology; Music therapy; Children with autism

\begin{abstract}
Music therapy based on computer technology has now become a popular way of psychological disease treatment. The treatment's main medium is music signal. Send music signals through computer, and then pass it to psychological consultant to make him get some energy to change his state of body and mind. Music therapy in children with autism treatment also has a good effect. This article analyzes music therapy in children with autism treatment under computer technology. First of all, analyze the definition and methods of music therapy, secondly understand the cause and characteristics of the children with autism, and then give music therapy strategy.
\end{abstract}

In music therapy treatment based on computer technology, use digital audio technology, computer technology and MIDI technology and combine with electronic instruments like synthesizer and sampler as audio terminal. Integrate the above technology and tools, and follow certain principle laws like music acoustics, instrumentation, electronics and computer technology so as to lead to music therapy. Now it has become a new type of music application subject and has got well practice in multiple fields.

\section{MUSIC THERAPY CONCEPT AND METHOD ANALYSIS}

A. Concept of music therapy

Music therapy is a comprehensive interdisciplinary. It is also known as "music therapy" and is an emerging edge discipline. America is the earliest to set it a formal discipline. The name, music therapy, is translated from English. Music therapists and theorists have different definitions. General definition of music therapy takes music as a major means of treatment. It is a new edge discipline involving multiple subjects such as music, medicine and psychology for patients' rehabilitation. Dr. Burner thought connotation should have six elements: interpersonal interaction process, therapist, music elements, the function of music therapy, patients, and method and form of music therapy. While professor Gao Tian in Central Conservatory of Music in China said: "music therapy is an intervention process of a system. In this process, the therapist uses various forms of music experience, as well as treatment relations developed in the course of treatment, which is the power of treatment to help the object (patients) to achieve the purpose of rehabilitation. [1]" Different people had different explanation details for music therapy concept, but basically they all mentioned that music therapy needed music therapists instruction of multidisciplinary theory and method. With the help of music's special effect on body and mind, choose music treatment technology according to the plan and purpose and participate in all kinds of music activities or related activities generated by computer technology with patients so as to eliminate psychological barrier, alleviate negative mood and achieve the purpose of physical and mental health.

\section{B. Music therapy}

Therapists generally summarized three groups of music therapy in actual treatment process, combining patients' ability and treatment target and different treatment techniques and music activities content: acceptance type, recreation type and improvisation type. 1. Acceptance type music therapy is mainly to achieve therapeutic purposes by listening to all kinds of music. So it can 
also be called hearing method. Hearing also needs technology. Here listening to music and discussion, active listening and music appreciation and music sensory stimulation method and so on are mainly adopted. 2. In recreational music therapy, therapists need to guide the patient actively participate in singing, music playing and dancing. Also they can adapt the music works based on medical need, and achieve treatment through adapted singing and music playing, etc. Patients not only listen to music under the treatment, but also involve in music activities of voices or instruments under the therapist guide in person [2]. 3. Improvisational music therapy combines vocals and instruments and allow patients to freely express their emotions and feeling. Use nonverbal way is more practical for patients with language barriers to express feelings, which is to use simpler in improvisation music treatment free combat rhythmic musical instrument or a percussion instrument.

\section{THE ANALYSIS OF CHILDREN WITH AUTISM}

\section{A. The concept of children with autism}

Autistic disorder is called loneliness disorder. It belongs to a kind of comprehensive development obstacle. The disease most starts in the period of infants before the age of 3 and throughout the whole life, so it can also be called childhood autism, etc. Autism can be divided into children with autism and adult with autism according to age groups. Here children with autism are mainly studied in music critical, namely children within 12 years of age. After the birth of a baby, he will have awareness of the surrounding environment such as hearing, touch, and with his physiological mature, this awareness will be increased. For example, when a baby is facing the mother's pick-up, he will make meet posture, and also has obvious preferences to sound toys, etc. But children with autism in facing of these often show glassy-eyes and rigid conduct, etc. They can't use normal conversational language to communicate with people.

\section{$B$. The reason for children with autism}

Causes for autism are complex and no single. After persistent research of relevant scholars, the factors may cause autism are found. In detail, the first is genetic factor. Most scholars believe that congenital autism genetic factors and gene mutations are associated with major chromosome abnormalities, but all the gene research cannot correctly identify autism defect belonging specific chromosome and gene location, etc. Scientists and scholars in recent years' research found that in the family of children with autism, 20\% families have autism families such as mentally retarded and language barriers. Stephan led a team research and found in March 2006 that: when the subjects had CNTNAP2 gene mutation, tested children who showed autistic tendencies, thus confirmed the genetic basis of autism. American geneticist Michael Wenglor thought that if one of the twins was with autism, the other autism risk would be as high as $75 \%$. He also proposed that male offspring with autism had higher rates than female offspring [3]. Experimental study shows that autism only relates to genetic factors, but sometimes it is not very obvious in some cases or has no expression. Neurobiology is the second factor. Women infect with virus during pregnancy because of German measles or influenza, so the baby gets head damage in head development; human body metabolic abnormalities can cause brain cells dysfunction or disorder; pregnant women get perinatal infection; pregnant women pregnancy fetal distress type abortion can cause baby cerebral agenesis. Pregnant women premature delivery, difficult labor or infants' encephalitis, meningitis and other damage to the brain's biological factors increase the chances of autism. Thus we can conclude that autism is not because parents thoughtlessness, and is often caused by abnormal brain nerve. Mental health such as pressure and emotional state before conception and during pregnancy may cause autism. Third is environment factor. External environment factor mainly refers to children exposure to toxic chemicals, such as toxic gas in the air, toxic chemicals in food or polluted drinking water, etc.; it may also be heavy metal infections, and so on. Many scholars generally agree that social environment factor is induced genetic change and is the cause of autism. Invisible genes here can be compared to loaded explosive bomb, and environmental factors are igniting fuses. Once the fuse is triggered, it could cause autism. Mostly children acquire autism by parental nurturing attitude. Parents' indifferent emotion, beat and scold are the fuses [4]. 


\title{
III. MUSIC THERAPY STRATEGY ANALYSIS IN CHILDREN WITH AUTISM TREATMENT BASED ON COMPUTER TECHNOLOGY
}

\author{
A. Guide the autistic children to establish a positive connection with the surrounding \\ environment
}

The world of children with autism is over self-closed. In the way of thinking and behavior ability, they are also different from normal people, and it is difficult to develop normal contact with the outside world and it is difficult to have normal communication, or live in harmony with others in society. Music therapy based on computer technology can lead these children with autism as far as possible to establish the contact with the environment so as to enhance their adaptation to the social environment. For example, when autistic children receive music therapy in treatment room, they usually do not notice indoor environment or even completely ignore them, but they will listen to a certain degree of music, respond to a sound, so in the process of early treatment, with the help of establishing connection with sound, just start with simple instruments, and then slowly expand simple instrument to other environmental objects associated with them. We need to use computer technology to make some music software. For example, make eight sections simple melody, and then continuously make repeated playing. For audio rendering, use two tones: angle iron and timpani. Use alternate presentation and handle the volume of the two instruments. Make angle iron volume relatively weak and timpani volume stronger. Before leading the autistic children into therapy, angle iron and drum are placed in front of the fixed position. After the autistic children get into the treatment room, make them still sit in the original position. Then play audio, and there are different tones and the same melody. The volumes of the two instruments are set to take the form of alternating, which will make children more intently listen to music. Therapists repeatedly play music for several times, and then they can go to the children side. When there is an angle iron melody, gently play angle iron; when there is a drum sound, the therapists heavily hit the drum. According to the audio, repeat play the instruments accordingly, then children will turn back to watch the therapist play. Therapists then stop playing and come back to the front of the computer and still play sound, and then children will stare at the instrument for a period of time, and then turn to the direction of the computer. The attention is still on the sound, but they have no desire and sign to play [5]. After a period of time, the therapist play the music again for a long time, and the therapist repeats the first action, and then children are finally starting to imitate the therapist to play the two instruments, and begin to establish connections with objects outside of sound environment.

$B$. Train autistic children language with the aid of computer music technology

Facing autistic children with serious language disorder, music therapists should slightly adjust the sound velocity and volume, enable children to have in-depth listening, and let the children learn self-behavior control in the process of listening. Therapists next take a variety of different tone with a combination of a variety of onomatopoetic words and guide children hum following music melody. This not only extends the richness of autistic children language, but also breaks the past well absolute quiet state and makes them hum in the music. Therapist can make use of computer technology to produce rich variety voice quality software and set up simple sound operation on a computer, and adjust the different timbre according to the actual circumstance of patients and treatment need [6].

C. Improve autistic children stereotyped behavior by music training under the use of computer technology

Behavior impairment is typical of children with autism. Children often show stereotyped behavior, and it is very difficult to change this behavior. The focus of therapist music therapy is to improve the repetitive patterns of behavior. The therapist can get ready a music production sound in the computer software, and the therapist may demonstrate jump leg movement while raising the pitch of the music melody, and make squat movement in reducing the pitch of a melody on the basis of original movement. Children will be very excited to imitate their actions. Then continue to broadcast the tone melody after the change, and the therapists should determine children psychological state. Tap on a different instrument with corresponding to the music and show children lightweight flexible arm movements to make children quickly attracted and imitate the 
action.

\section{CONCLUSION}

Music treatment in children with autism demands higher, especially there are very high requirements for music therapists. Music therapy in children with autism is very flexible, and there is no specific treatment. Therapists need to do more observation and analysis in the process of treatment, and adjust and guide according to children reaction. Music therapy with computer technology can meet the demands, and music software with computer technology can be adjusted in the process of treatment, and the treatment efficiency and effect are excellent.

\section{REFERENCES}

[1] Hui Lin. The influence research of music for the treatment of children with autism [D]. Yanbian University, 2014.

[2] Huang Mujun. Orff music therapy application research in cases of children with autism [D]. East China Normal University, 2014.

[3] Liu Yingman. The investigations of music education influence on the body and mind of children with autism [D]. Hebei Normal University, 2010.

[4] Wang Ye. Introduction to the application of computer music in children with autism treatment [J]. Journal of Music Observatory, 2013, 12:235-236.

[5] Cao Lingyun. Improvisation music therapy research for the social interaction ability of children with autism [D]. The results of East China Normal University, 2009.

[6] Wang Ye, Lian Yun. Computer music production in music therapy in children with autism [J]. Hundreds of Art, 2011, S2:423-424. 